Research Article

\title{
LRP Model and Algorithm of Expressway Logistics Network Planning Based on Internet of Things
}

\author{
Zheng Kou $\mathbb{i D}^{1}$ and Man Zhang $\mathbb{D}^{2}$ \\ ${ }^{1}$ College of Business, Yancheng Teachers University, Yancheng 224007, Jiangsu, China \\ ${ }^{2}$ Economic and Trade Department, Yancheng Polytechnic College, Yancheng 224005, Jiangsu, China \\ Correspondence should be addressed to Man Zhang; zhangman8@hotmail.com
}

Received 13 July 2021; Revised 9 August 2021; Accepted 7 September 2021; Published 20 September 2021

Academic Editor: Sang-Bing Tsai

Copyright ( $) 2021$ Zheng Kou and Man Zhang. This is an open access article distributed under the Creative Commons Attribution License, which permits unrestricted use, distribution, and reproduction in any medium, provided the original work is properly cited.

\begin{abstract}
With the continuous improvement of the expressway logistics network, the location-routing problems (LRP) have become the obstacle to be overcome in the development of related industries. Based on the needs of modernization, in the era of the Internet of Things, classic traffic path planning algorithms can no longer meet the increasingly diverse needs, and related research results are not ideal. To reduce logistics costs and meet customer needs, this paper studies transportation route planning models and algorithms based on Internet of Things technology and particle swarm optimization. Firstly, the LRP model of expressway logistics network planning analyzes the achievement of goals, lists the assumptions, and builds the LRP model of expressway logistics network planning based on the mathematical model of path planning. Then the model is optimized and solved based on the particle swarm optimization algorithm. In order to verify the effectiveness and feasibility of the algorithm, MATLAB is used to simulate the algorithm. Finally, the LRP particle swarm optimization model of highway logistics network planning is put into the actual distribution work of a logistics company to analyze the change of distribution cost and investigate the related satisfaction. Experimental data show that the improved particle swarm optimization algorithm in this paper begins to converge in the 100 th generation, the shortest running time is $57 \mathrm{~s}$, and the value of the objective function fluctuates slightly around 880 . This shows that the model algorithm in this paper has strong search ability and stability. In the simulation experiment, the optimal objective function value of the model is 1001 yuan, which can be used to formulate the optimal distribution scheme. In the actual distribution work, the total cost of distribution before and after the application of the model was 12176.99 yuan and 9978.4 yuan, the fuel consumption cost decreased by 2097.23 yuan, and the penalty cost decreased by $85 \%$. In the satisfaction survey, the satisfaction of all people was 9 points or above, with an average score of 9.42 points. This shows that the LRP particle swarm optimization model of expressway logistics network planning based on the Internet of Things technology can effectively save distribution costs and improve satisfaction.
\end{abstract}

\section{Introduction}

1.1. Background Significance. On a global scale, the Internet of Things technology is not only the breakthrough point and key development direction of future information technology, but also one of the important engines leading the full recovery of the global economy. With the continuous development of the industrialization of the Internet of Things, it has been widely used in various fields and has brought a great impact on people's production and life [1]. Expressway has the economic and technical characteristics of high speed, large capacity, low transportation cost, safe and comfortable driving, which is conducive to the centralized use of land resources, the continuous reduction of energy consumption, the reduction of environmental pollution, the stable improvement of traffic safety, and the realization of sustainable development [2]. Combining the Internet of Things technology and the formed highway network, developing logistics network and solving the problem of transportation path planning is a new idea, which can better meet the increasingly diversified logistics needs. 
1.2. Related Work. Internet of Things technology has been the focus of research in various fields since its birth. Referring to the logic of service-oriented, Balaji proposed that the interaction between customers and IOT retail technology would lead to the cocreation of value. He collected responses from 289 IOT technology users in the retail environment [3]. According to the functional requirements of carbon emission management system, Bo, combined with radio frequency identification technology and wireless sensor network, studied the basic principle and structure of port logistics system [4]. He designed the hardware of the system aiming at intelligent node, gateway node, and operating system and tested the system on site. The importance of path planning in the field of logistics distribution is self-evident, so many researchers have analyzed it. For fresh e-commerce enterprises in the low-carbon emission environment, Guo proposed a forward/reverse twostage logistics network and path planning model for the first time (the goal of the first stage is to minimize the overall cost of the system, and the second stage is to minimize the total cost of the distribution vehicle routing) [5]. The validity of the model is verified by taking fresh food e-commerce enterprises as the research object. Based on the multiobjective mixed integer programming method, Hao proposed an improved mathematical formula. The model considered the system operation cost and the risk of local residents and introduced a compensation factor into the risk objective function [6]. After that, he coded and calculated in LINGO optimization solver and used the augmented $\varepsilon$-constraint method to generate Pareto optimal curve of multiobjective optimization problem. His method is innovative, but the data set used in simulation experiment is not representative.

1.3. Innovative Points in This Paper. In order to improve the efficiency of logistics distribution, reduce costs, and better meet the needs of customers, this paper combines the Internet of Things technology and the formed highway network to solve the complex transportation path planning problem. The innovation points of this study are as follows: (1) Firstly, the LRP model of expressway logistics network planning is constructed after analyzing the model objectives, and then the model is optimized and solved with particle swarm optimization algorithm. (2) The simulation experiment with MATLAB proves that the model algorithm in this paper has strong search ability and stability. (3) The LRP particle swarm optimization model of highway logistics network planning is applied to the actual distribution work of a logistics company. The change of distribution cost before and after application is analyzed, and the satisfaction of employees, customers, and drivers is investigated.

\section{Internet of Things Technology and LRP Model of Expressway Logistics Network Planning}

\subsection{Internet of Things Related Technologies}

2.1.1. Types of Internet of Things Technology. The core technologies of the Internet of Things includes radio frequency identification technology, barcode technology, communication technology, and remote sensing technology. The Internet of Things is an aggregation technology, based on the Internet and mobile communication networks, using smart objects to connect independent physical objects, with strong processing, reliability, and comprehensiveness [7, 8].

Ubiquitous sensing technology is the foundation, which can perceive information through the perception equipment and use the network to obtain relevant information and mine the information, so as to transform the massive complex data information into useful information that people need. Radio frequency identification (RFID) technology and global positioning system (GPS) are widely used in the field of logistics. GPS can sense the moving objects and realize the intelligent and visualization of logistics distribution.

In the process of data transmission, the technology will analyze and process the data using an intelligent computing and processing system. Information aggregation technology will use multiple sensors to detect targets at the same time, obtain multiangle information, and then fuse them to improve the accuracy of data. Therefore, it embodies the integration of data collection, storage, transmission, and extraction functions $[9,10]$. The core of information aggregation is to analyze and process multisource information, and its basis is multisensor.

Lean computing technology is based on big data and integrates cloud computing, data mining and multimedia interactive processing and other related technologies in the Internet environment. The computing resources of cloud computing are dynamic and can be provided in the form of services. Data mining needs to use certain mining technology to find out the potential relationship between data.

2.1.2. Cognitive Model of Internet of Things Technology. The cognitive form of Internet of Things technology is based on the "memory computing" mode. It adapts to the occurrence mechanism of human brain cognition and is related to data mining, memory, analysis, and other technologies. From the perspective of cognition, technology cognition is a corresponding cognitive form realized by Internet of Things technology. Cognitive function reflects the value of technology application. On the one hand, the information and data resources obtained by sensors are transferred to the data center for storage, so as to build a huge database resource. Like the storage function of human brain, it can store enough information for analysis. On the other hand, the mapping relationship is established from various functional relations and corresponding relations of model data preservation, and the relationship between form, content, and connotation is established. The results required by the feedback operation and processing are applied to the next operation and processing process of the system by powerful computer.

Cognitive form mainly depends on the technologies in the Internet of Things and has the technical characteristics of accuracy, quantification, and high efficiency. The technology system of Internet of Things displays the state of things through information data, analyzes people's intention, and 
interacts with and controls the outside world through sensing and somatosensory technology. The cognitive process is analyzed with precision, information, and intelligent technology to realize the quantitative control of technology, which provides an important reference for the preliminary judgment of analysis.

The cognitive process of the technology system of the Internet of Things adapts to the cognitive form of the human brain through the integration of sensor, data storage, calculation and analysis, program function setting, and other technologies and theories to create a cognitive system of technology $[11,12]$. This is done through the sensor instead of human senses to obtain information, literacy, reprocessing for storage, calculation under the specific running program and code function, and feedback to the application field, to achieve the purpose of intelligent cognition.

\subsubsection{Characteristics of Internet of Things Technology} Cognition. Through accurate and intelligent analysis and comparison of cyberspace, calculating reasonable and economical resources, and effectively configuring the actual operation and physical resources of the Internet of Things technology control system, the cognitive process is based on information. Through virtual information simulation scenarios, use a small amount of material resources informatization to meet the existing production and life needs of the system [13]. Visual information and perception technology can realize intelligent and efficient control and improve the efficiency of processing and analyzing information.

The information under the cognition of the Internet of Things has the characteristics of interactive feedback. The interaction is between the client and the external environment, between the system network and the application platform, and between each object through the data system, establishing a wide range of physical relations in the information way. Quantitative control can improve the certainty of the development of things, grasp the law of development, and improve the accuracy of prediction.

The control factors in cognitive process include reliability, stability, information security, and privacy protection $[3,14]$. Relying on the virtual network information platform, the Internet of Things platform becomes the basis of the flow of physical resources such as material, energy, and information and realizes various applications on this basis. Data storage and computing technology plays an important role in the Internet of Things system. In the mature development process, the continuously improved cloud computing and cloud storage technologies are used for information and intelligent and automatic computing processing. It has certain cognitive function, structure, cognition, and control and execution functions.

\subsection{Expressway Logistics Network Planning}

2.2.1. Highway Transportation Network and Logistics Network. The logistics network is a dynamic network that provides freight services to customer nodes at the lowest cost and accurate arrival time through the layout of logistics nodes, the arrangement of transportation routes between logistics nodes, and the selection of transportation means. The expressway traffic network is mainly composed of its own routes and nodes and is a static network of traffic infrastructure that provides trunk services.

Due to the connection of expressway service area with expressway, it is very convenient to pick up and load vehicles, and it has the advantages of fast and convenient logistics transportation and distribution [15]. The expressway service area can also provide logistics services to the surrounding cities and produce a certain scale of logistics value-added services, which has the advantages of logistics radiation effect.

Third-party logistics enterprises organize certain transportation methods on the basis of highway transportation network, supervise the planning and operation management of logistics transportation, and construct logistics transportation channel by expressway. It can reduce the cost of logistics transportation, improve the efficiency of logistics transportation, promote the formation of comprehensive transportation system, and improve the capacity of regional transportation.

\subsubsection{Influencing Factors of Logistics Network Planning.} Whether an enterprise can accurately predict market demand, whether it can afford to build a logistics distribution center and its operating costs, and whether it can supply and distribute sufficient products in time will have a certain impact on the planning of logistics distribution network [16]. Moreover, these elements complement each other. Only when these factors are combined and fully considered and analyzed can enterprises plan and build the most suitable logistics distribution network for their own development.

The real good logistics distribution network layout and processing capacity must be consistent with the market demand. Logistics distribution network planning needs to consider all kinds of costs in the process of construction and operation. Generally speaking, the ultimate goal of network planning is to reduce the total cost. This cost includes the cost of logistics distribution facilities (such as vehicles and packaging boxes), ordering cost, cargo transportation cost, inventory cost, and management cost [17].

The stronger the supply capacity of logistics distribution network is, the better it can meet the needs of customers. Generally awesome supply capacity, order completion rate, and replenishment cycle supply level are used as indicators to measure the supply capacity of logistics distribution network [18]. In the competitive market environment, the efficiency of logistics distribution is one of the important factors for enterprises to attract customers and expand market share. High efficiency of logistics distribution needs to set up more logistics distribution centers, which may increase the distribution cost. Therefore, in the planning, we should set up a reasonable logistics distribution efficiency.

2.2.3. Technology Involved in Expressway Logistics Network Planning. The location of logistics nodes should be based on the overall logistics network and social and economic 
benefits and comprehensively consider the number, specific location, construction cost, and functional positioning of logistics nodes. To develop logistics nodes on expressways, first consider the location of the logistics network. Different location and layout schemes have different operating efficiency and economic effects [19]. After the location is determined, it is necessary to divide the service scope of logistics nodes. Suppose there are $x$ logistics nodes, which are $w_{1}, w_{2}, \ldots, w_{x}$, respectively. The shortest distance between any two logistics nodes is $d_{i j}$ and its radius is $G_{i j}$. There are $y$ customer nodes, which are $k_{1}, k_{2}, \ldots, k_{y}$, respectively. The distance between any logistics node $w_{i}$ and customer node $k_{u}$ is $g_{i u}$.

$$
\begin{cases}k_{u} \in w_{i}, & \text { if } g_{i u}<\min \left\{G_{i j}\right\}, \\ k_{u} \notin w_{i}, & \text { otherwise, }\end{cases}
$$

where $\min \left\{G_{i j}\right\}$ is the service scope of the logistics node. If there is a customer node $k_{1}, k_{2}, \ldots, k_{t}, k_{y}$ in the cluster summary of logistics node $w_{i}$, formula (2) is satisfied:

$$
\sum_{u=1}^{t} q_{u}=x C_{a}
$$

Then, logistics node $w_{i}$ will serve customer node $k_{1}, k_{2}, \ldots, k_{t}, k_{y}$, where $q_{u}$ is the logistics demand of the customer node and $C_{a}$ is the capacity of the transport vehicle. In the planning of expressway logistics network, it is also necessary to consider the arrangement of vehicles, including the type and quantity of vehicles [20, 21]. Avoid the waste of transportation resources and costs caused by unreasonable transportation routes, ensure the punctuality of logistics, and meet the time demand.

\subsection{Location-Routing Problems (LRP) Model}

2.3.1. Classification of LRP. Through known and unknown customer demand and vehicle load, location routing problem (LRP) can be divided into determinism and randomness. According to the number of available distribution centers, it can be divided into single center and multiple centers. According to the available models and the maximum load capacity, it can be divided into single models and multiple models. The maximum load capacity of bicycles is the same, but the maximum load capacity of many models is different.

According to whether the carrying capacity will exceed the capacity or the maximum carrying capacity of the vehicle, it can be divided into deterministic type and uncertain type [22]. According to whether the distribution center is the starting point of transportation, it can be divided into terminal type and medium transition type [23]. If the whole period is one or more time periods, it can be divided into static and dynamic.

As for the customer's requirements of receiving time, it can be divided into time limit and no time limit. As for the number of objective functions, it can be divided into single objective and multiobjective. The source of data can be divided into random and practical problems. Some data in random problems are difficult to obtain, so some assumptions are needed, while the data of actual problems can be directly obtained [24].

2.3.2. Mathematical Model of LRP. The mathematical model of path planning includes objective function and constraint conditions. Suppose there are $x$ customer points, $y$ distribution centers, and $\mathrm{K}$ vehicles. The objective functions of vehicles arriving within the time required by customers, the lowest total cost, and the shortest driving path are shown in

$$
\begin{aligned}
& \min \sum_{b=1}^{x}\left\{\max \left[\left(F T_{b}-T_{b}\right), 0\right]+\max \left[\left(T_{b}-M T_{b}\right), 0\right]\right\} \\
& \min \sum_{k=1}^{K} D_{k} \sum_{a=1}^{x+y} \sum_{b=1}^{x} R_{a b k} p_{b}+\sum_{k=1}^{K} \sum_{a=1}^{x+y} \sum_{b=1}^{x+y} D_{a b k} P_{b} e_{a b}, \\
& \min \sum_{k=1}^{K} \sum_{a=1}^{x+y} \sum_{b=1}^{x+y} R_{a b k} e_{a b}
\end{aligned}
$$

where $D_{k}$ is the loading cost of the $k$ th vehicle; $D_{a b k}$ is the freight of the $k$ th car from $a$ to $b ; P_{b}$ is the quantity of goods to be delivered to point $b ; e_{a b}$ is the distance from $a$ to $b ; R_{a b k}$ is the decision variable; $F T_{b}, M T_{b}$ are the starting and ending points of the arrival time required by customers; $T_{b}$ is the estimated time of goods delivery.

The constraints are that each arrival point uses only one vehicle to deliver goods, each vehicle cannot exceed the loading capacity, and each vehicle can only have one distribution center as shown in

$$
\begin{gathered}
\sum_{k=1}^{K} \sum_{a=1}^{x+y} R_{a b k}=1, \\
\sum_{a=1}^{x+y} \sum_{b=1}^{x} R_{a b k} p_{b} \leq z_{k}, \\
\sum_{a=x+1}^{x+y} \sum_{b=1}^{x} R_{a b k} \leq 1,
\end{gathered}
$$

where $z_{k}$ is the loading capacity of the $k$ th vehicle. The constraints to ensure the continuity of the route and the time sequence of the driving route are shown in formulas (9) and (10), respectively:

$$
\begin{array}{r}
\sum_{a=1}^{x+y} R_{a p k}-\sum_{b=1}^{x+y} R_{p b k}=0, \\
R_{a b k}\left(T_{b}-T_{a}\right) \geq 0 .
\end{array}
$$

It means that each distribution center has a car to leave, the vehicle will not leave from an open distribution center, any two distribution centers are not connected, and any two distribution centers are not on the same route. The constraints are shown in formulas (11)-(14) respectively: 


$$
\begin{array}{r}
\sum_{b=1}^{x} \sum_{k=1}^{K} R_{a b k}-s_{a} \geq 0, \\
\sum_{b=1}^{x} R_{a b k}-s_{a} \leq 0, \\
\sum_{k=1}^{K} R_{a b k}+s_{a}+s_{b} \leq 2, \\
\sum_{a=x+1}^{x+y} \sum_{p=1}^{x} R_{a p k}+\sum_{a=1}^{x} \sum_{b=x+1}^{x+y} R_{a b k} \leq 1 .
\end{array}
$$

Among them, $s_{a}, s_{b}$ is the decision variable, which indicates whether the distribution center in this decision is open or not.

2.3.3. Algorithm of LRP Model. Precise algorithm and heuristic algorithm can be used in solving LRP model. Precise algorithms include integer programming, branch and bound, dynamic programming, and nonlinear programming [25]. However, as the complexity of LRP becomes higher and higher, the precise algorithm cannot solve the large-scale problems. Therefore, the heuristic algorithm for solving optimization problems is generally used to solve LRP [26].

Particle swarm optimization (PSO) simulates the foraging behavior of birds. There are $n$ particles in the $c$-dimensional search space, which are $r_{i}=\left(r_{i 1}, r_{i 2}, \ldots, r_{i c}\right), i=1,2, \ldots, n$ and fly in the solution space at the speed of $s_{i}=\left(s_{i 1}, s_{i 2}, \ldots, s_{i c}\right), i=1,2, \ldots, n$. Then, the calculation of particle velocity and displacement is shown in formulas (15) and (16), respectively:

$$
\begin{aligned}
s_{i j}(x+1)= & w s_{i j}(x)+c_{1} j_{1}\left(p_{i j}(x)-r_{i j}(x)\right) \\
& +c_{2} j_{2}\left(p_{g i}(x)-r_{i j}(x)\right), \\
r_{i j}(x+1)= & r_{i j}(x)+s_{i j}(x+1) .
\end{aligned}
$$

Among them, $c_{1}, c_{2}$ are self-factor adjusted particles flying to their best position and global factor adjusted particles flying to the global optimal position respectively. $j_{1}, j_{2}$ are random numbers between 0 and 1 . In order to simplify the calculation, the analysis of convergence can be simplified to study the behavior of a single particle. Therefore, the calculation of particle velocity and displacement can be simplified as

$$
\begin{aligned}
& s(x+1)=w s(x)+\delta(z-r(x)), \\
& r(x+1)=r(x)+s(x) .
\end{aligned}
$$

By substituting formula (18) into (17), we can calculate the recurrence relationship of velocity iteration formula in $x, x+1, x+2$ time periods:

$$
s(x+2)+(\delta-1-w) s(x+1)+w s(x)=0 .
$$

Formulas (17) and (18) can be expressed in the form of matrix:

$$
y(x+2)=a y(x+1)+b p
$$

where $a, b, p$ represent the dynamic behavior of particles, the input matrix, and the external forces driving the particles to fly, respectively.

\section{Experiments on LRP Model Construction and Optimization Algorithm for Expressway Logistics Network Planning}

\subsection{Construction of LRP Model for Expressway Logistics Network Planning}

3.1.1. Objectives of Model Building. Highway logistics network planning LRP is a multiobjective optimization problem. The goals to be met include using the highway logistics network to plan, reasonably arrange vehicle routes, shorten the total distribution path, and reduce vehicle costs. Improving the quality of logistics services and customer satisfaction aims to meet customer demand for delivery time, realize the lowest total cost of distribution, and bring high benefits for distribution companies.

3.1.2. Model Hypothesis and Construction. There are many distribution centers, and the number of vehicles in each distribution center is enough, and the models are consistent. The total demand of each transport route does not exceed the maximum loading capacity of the vehicle. Each customer has its own requirements on the delivery time of the goods. Each customer has only one distribution center, the loading and unloading time is consistent, and the distribution of goods is consistent.

Based on the above objectives and assumptions, combined with the LRP mathematical model in the second chapter of this paper, the LRP model of highway logistics network planning is constructed.

\subsection{Solving Model of Particle Swarm Optimization Algorithm}

3.2.1. Particle Coding. The PSO algorithm has problems such as premature convergence, dimensionality disaster, and easiness of falling into local extreme values, which need to be solved. According to the principles of nonredundancy, soundness, and completeness, natural number coding is used to solve vehicle routing problems and sequence problems. Each particle is divided into three segments. The first random natural number corresponds to the vehicle number, the second random natural number corresponds to each customer's service order, and the third random natural number corresponds to the vehicle distribution center number.

3.2.2. Calculation of Vehicle Number and Fitness Function. The calculation of the number of vehicles required to complete a distribution task is shown in 


$$
N=\left[\sum_{u=1}^{M} \frac{x_{u}}{r}\right],
$$

where $r, x_{u}$ represent the capacity of the car and the demand of customers, respectively. According to the actual situation, the number of vehicles is rounded up.

By integrating the objective functions in the LRP mathematical model, the fitness function of the LRP model is obtained:

$$
Z=\frac{1}{\left(F_{1}+F_{2}\right)},
$$

where $F_{1}, F_{2}$ are distribution time function and distribution center cost function, respectively.

3.2.3. Mutation Operation of Particles. In order to improve the quality of the solution and avoid local optimization, mutation operation is needed for particles. Two variants are randomly generated to exchange the particles in corresponding positions.

3.3. Case Simulation and Practical Application. In order to verify the effectiveness and feasibility of particle swarm optimization algorithm in LRP model of highway logistics network planning, MATLAB is used to carry out simulation experiments.

A logistics company plans to establish its own distribution network on the basis of highway logistics network planning. The company has 3 distribution centers and 15 customer points that need to distribute goods. The operating cost of each distribution center is 100 yuan. The distribution of distribution centers and customers is shown in Figure 1.

The fixed cost of each departure is 120 yuan, and the unit distance transportation cost is 0.8 yuan per kilometer. It is assumed that the vehicle runs at an average speed, and the speed is controlled at $35 \mathrm{~km} / \mathrm{h}$. If the vehicle arrives earlier than the expected time window, it needs to pay 0.3 yuan per minute; if the vehicle arrives later than the expected time window, it needs to pay 0.5 yuan per minute.

The main purpose is to analyze changes in distribution costs, investigate customer satisfaction, and provide directions for further improvement of the model. Although simulation experiments can better simulate logistics and distribution, they cannot get customer feedback. Therefore, through simulation experiments, the LRP particle swarm optimization model of highway logistics network planning is applied to the actual distribution work of a logistics company.

\section{Discussion on Evaluation of LRP Model for Expressway Logistics Network Planning}

4.1. Performance Analysis of the Algorithm. The convergence diagrams of the three algorithms are shown in the figure: in order to verify the effectiveness and feasibility of the improved particle swarm optimization algorithm, the number of iterations is set to 300 , and the improved particle swarm

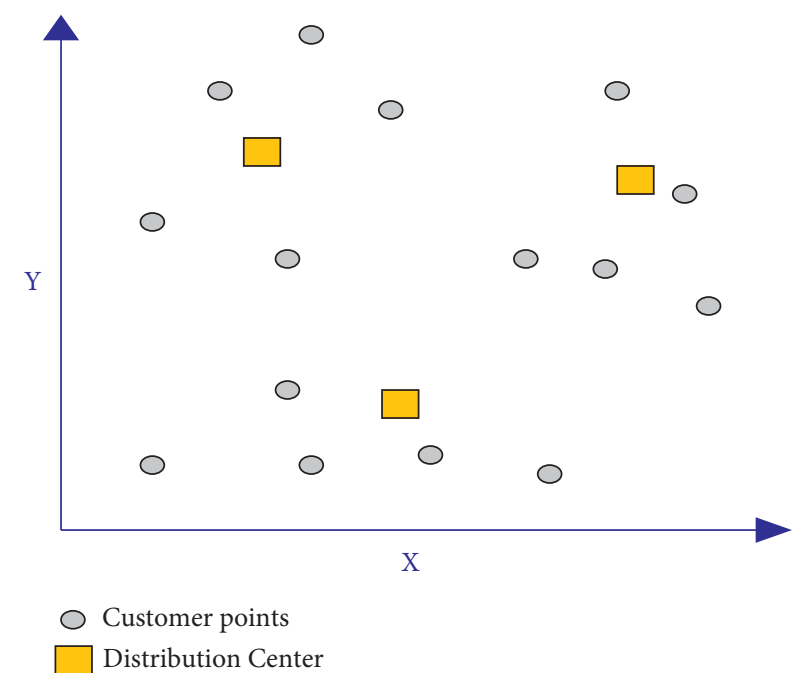

FIgURE 1: Distribution center and customer distribution.

optimization algorithm, standard particle swarm optimization algorithm, and genetic algorithm are given, respectively. The optimal solution result and the average running time are compared.

As shown in Figure 2, there are differences in the convergence speed of the three algorithms. In this paper, the improved particle swarm optimization algorithm began to converge in the 100th generation, the genetic algorithm began to converge in the 150th generation, and the standard particle swarm optimization algorithm began to converge in the 160th generation. This shows that the improved particle swarm optimization algorithm is better than genetic algorithm and standard particle swarm optimization algorithm in convergence speed.

Then, the maximum iteration number of the program is set to 1500 generations, and the three algorithms are run 10 times respectively. The running results of LRP model are as follows.

As shown in Table 1, the running time and target value of different algorithms are different in different running times. The optimal objective function value is 801 yuan, which is the result of the third run of the improved particle swarm optimization algorithm. The running time of the three algorithms in each run is compared.

As shown in Figure 3, the running time of the improved PSO algorithm in each run is shorter than the other two algorithms, and the shortest is $57 \mathrm{~s}$ in the third run. This shows that under the same running condition, the running time of the improved PSO is shorter. Since the algorithm has the characteristics of random search, the stability will be affected to some extent. Therefore, it is necessary to analyze the change trend of the ten order objective function values of the three algorithms.

As shown in Figure 4, the change of the objective function value of the improved particle swarm optimization algorithm is relatively stable, floating around the average value of 880 . However, the changes of the other two algorithms fluctuate, and the differences between the maximum and minimum values are 159 and 176, respectively. This 


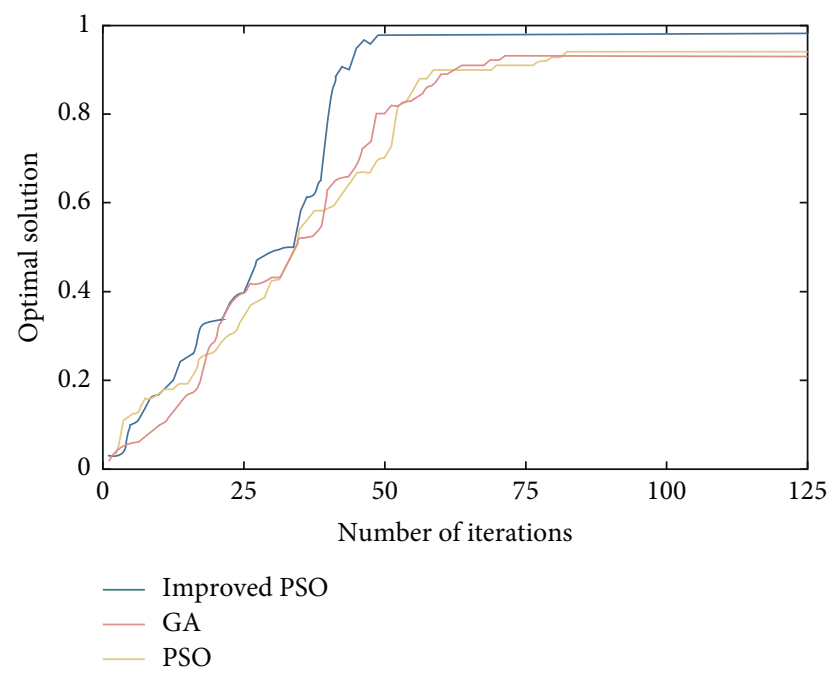

FIgUre 2: Performance analysis of the algorithm.

TABLE 1: Results of ten runs of three algorithms.

\begin{tabular}{|c|c|c|c|c|c|c|}
\hline \multirow{2}{*}{ Number of runs } & \multicolumn{2}{|c|}{ Improved PSO } & \multicolumn{2}{|c|}{ GA } & \multicolumn{2}{|c|}{$\mathrm{PSO}$} \\
\hline & Time (s) & Value & Time (s) & Value & Time (s) & Value \\
\hline 1 & 59 & 856 & 86 & 1051 & 98 & 1265 \\
\hline 2 & 62 & 869 & 93 & 1093 & 115 & 1287 \\
\hline 3 & 57 & 801 & 101 & 1114 & 123 & 1321 \\
\hline 4 & 63 & 873 & 88 & 1072 & 98 & 1291 \\
\hline 5 & 65 & 901 & 83 & 998 & 99 & 1313 \\
\hline 6 & 64 & 887 & 94 & 1102 & 105 & 1364 \\
\hline 7 & 65 & 893 & 99 & 1125 & 111 & 1377 \\
\hline 8 & 71 & 913 & 85 & 1021 & 95 & 1201 \\
\hline 9 & 69 & 905 & 97 & 1157 & 97 & 1252 \\
\hline 10 & 68 & 904 & 95 & 1099 & 108 & 1304 \\
\hline
\end{tabular}

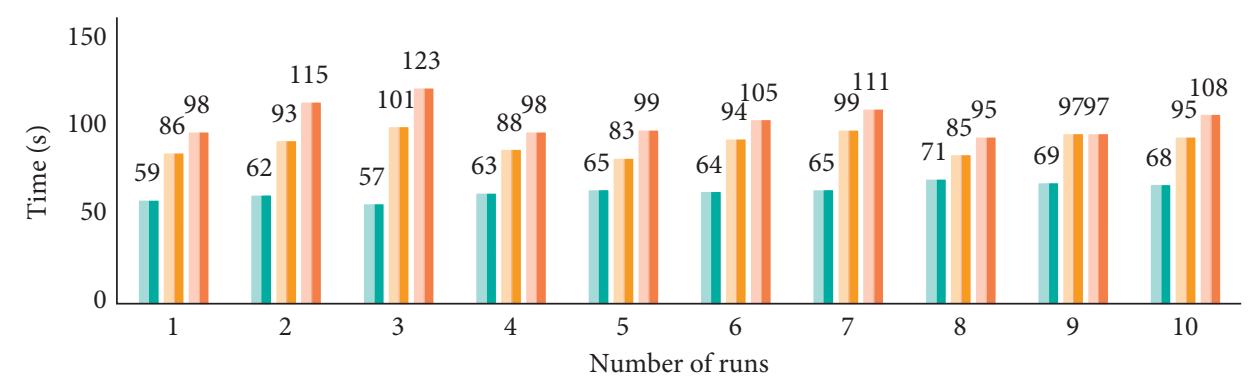

1. Improved PSO

1. GA

II PSO

Figure 3: Running time of different algorithms.

shows that the improved particle swarm optimization algorithm has high stability.

4.2. Model Case Simulation Results. In the simulation case, the goods and time requirements of 15 customer points are as follows.
As shown in Table 2, due to time constraints, the number of customers in this experiment is eight. Each customer site has its own cargo and time requirements. According to the customer's start time and end time, calculating the sensitivity of each customer's time demand, the goods must be delivered within the expected time window; otherwise, it will be necessary to compensate for the increased delivery cost. 


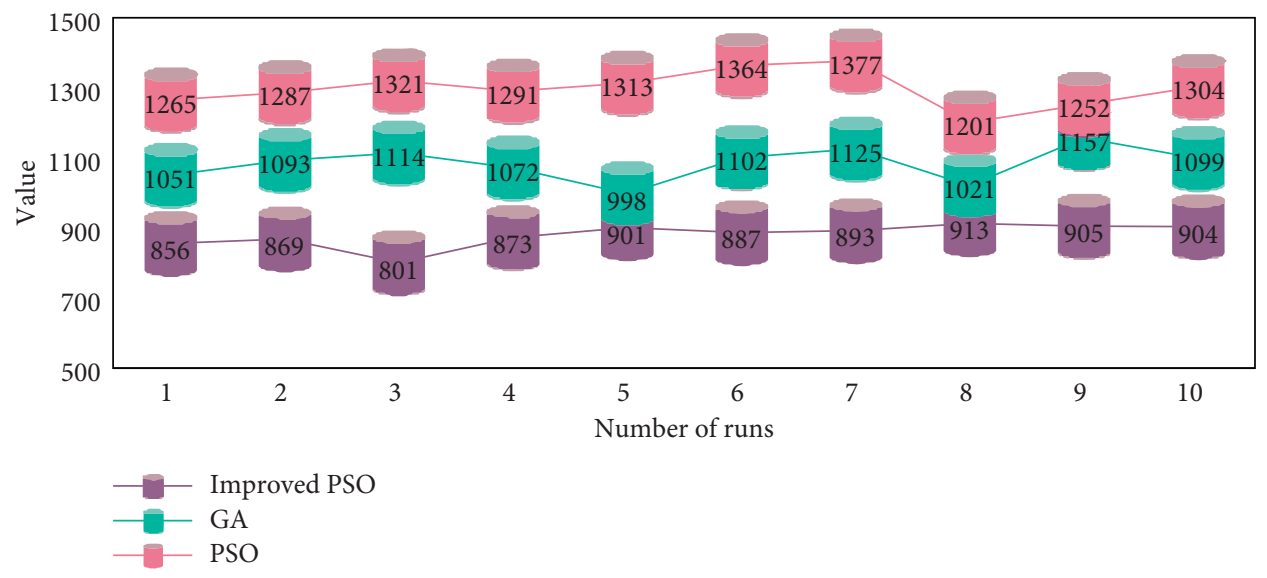

FIgURE 4: Change trend of objective function value.

TABLE 2: Goods and time requirements of customer points.

\begin{tabular}{lccccccc}
\hline Client & 1 & 2 & 3 & 4 & 5 & 6 & 7 \\
Distribution volume & 15 & 60 & 30 & 30 & 35 & 40 & 40 \\
Start time & 3 & 5 & 6 & 12 & 13 & 5 & 50 \\
End time & 101 & 125 & 136 & 105 & 150 & 117 & 12 \\
Client & 9 & 10 & 11 & 12 & 13 & 14 & 13 \\
Distribution volume & 155 & 45 & 50 & 20 & 35 & 40 & 55 \\
Start time & 5 & 6 & 3 & 12 & 13 & 6 & - \\
End time & 103 & 127 & 111 & 115 & 108 & 117 & 114 \\
\hline
\end{tabular}

As shown in Table 3, customer point 4 and customer point 13 are very time sensitive, and their sensitivity is 1 , while customer point 5 and customer point 12 are less sensitive to time. Therefore, it is necessary to plan the distribution scheme according to the customer's time sensitivity.

In order to meet the needs of goods and time of each customer point, the LRP particle swarm optimization model of expressway logistics network planning is run. The results of ten runs are as follows.

As shown in Figure 5, the optimal objective function value is 1001 yuan, and the corresponding distribution route and vehicle scheme are as follows.

As shown in Figure 6, each color line segment represents different vehicles. Each distribution center sends 2 vehicles, a total of 6 vehicles. Distribution center 1 sends vehicles A and B to customers 1-3-5 and 2-4 respectively. Distribution center 2 sends vehicles $C$ and $D$ to 6-10-7-8 and 9 customer points respectively. Distribution center 3 sends vehicles $\mathrm{E}$ and $\mathrm{F}$ to $11-$ 14-13 customer points and 15-12 customer points respectively.

\subsection{Practical Application of LRP Particle Swarm Optimization Model in Expressway Logistics Network Planning}

4.3.1. Distribution Cost Comparison. The LRP particle swarm optimization model of highway logistics network planning is put into the actual work of a logistics company, and the daily distribution cost of a week before and after using the model is compared. This paper analyzes whether the model can effectively reduce the distribution cost in the
TABle 3: Customer time demand sensitivity.

\begin{tabular}{lcccccccc}
\hline Client & 1 & 2 & 3 & 4 & 5 & 6 & 7 & 8 \\
Sensitivity & 0.7 & 0.6 & 0.6 & 1 & 0.8 & 0.8 & 0.5 & 0.8 \\
Client & 9 & 10 & 11 & 12 & 13 & 14 & 15 & - \\
Sensitivity & 0.6 & 0.5 & 0.6 & 0.5 & 1 & 0.6 & 0.7 & - \\
\hline
\end{tabular}

actual distribution and create higher benefits for the logistics company.

As shown in Table 4, there is a big difference in the total daily distribution cost of a week before and after the application of the model. The total cost of distribution is 12176.99 yuan one week before the model is applied. After the model is applied, the distribution cost of one week is reduced to 9978.4 yuan. In order to compare them more intuitively, it is drawn as a bar chart.

As shown in Figure 7, the daily distribution cost after model application is lower than that before model application. Especially on the 7th day, the difference was 381.29 yuan. In the process of distribution, the distribution cost of each vehicle includes fixed cost and fuel consumption cost. If the vehicle arrives earlier or later than the expected time window, penalty cost should be paid. The proportion of distribution cost before and after the application of the model is analyzed in detail.

As shown in Table 5, the fixed cost remains unchanged before and after the model is applied, but the fuel consumption cost and penalty cost are greatly reduced. The application of the model makes the distribution routing scheme more scientific and intelligent. The fuel consumption cost is reduced by 2097.23 yuan, while the penalty cost is 


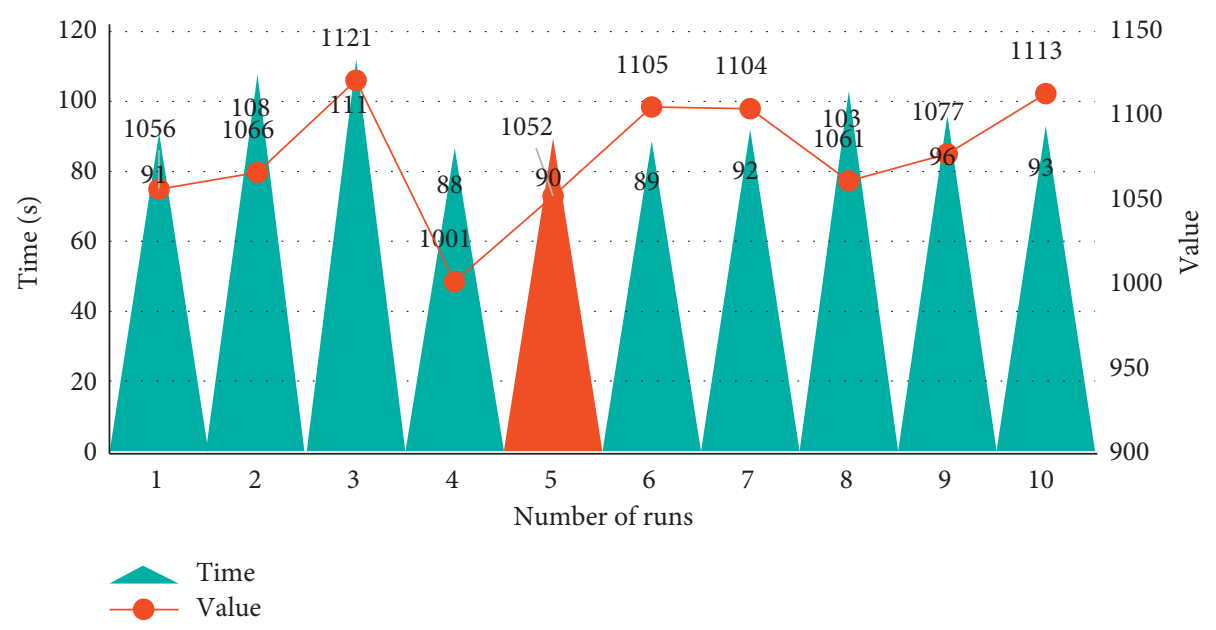

FIgURE 5: Results of ten runs of the model in this paper.

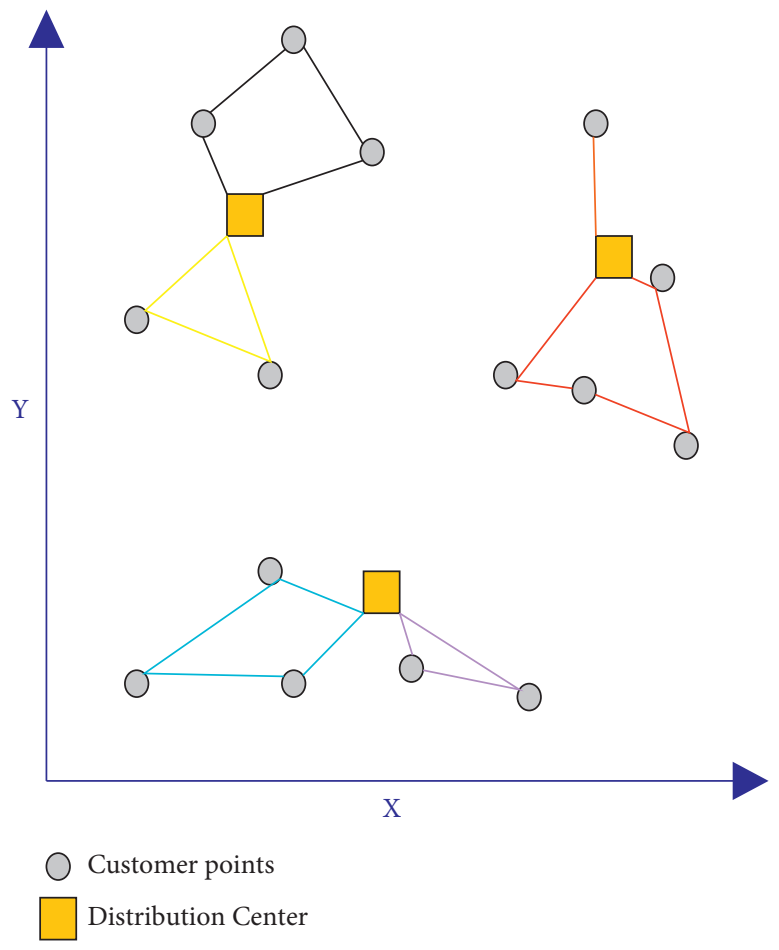

Figure 6: Optimal distribution route and vehicle scheme.

TABle 4: Comparison of daily distribution cost in a week before and after model application (yuan).

\begin{tabular}{lcc}
\hline Date & Cost before & Cost after \\
\hline 1 & 1745.57 & 1416.92 \\
2 & 1640.74 & 1427.74 \\
3 & 1751.78 & 1439.44 \\
4 & 1654.24 & 1387.14 \\
5 & 1755.08 & 1422.73 \\
6 & 1825.56 & 1461.7 \\
7 & 1804.02 & 1422.73 \\
Total & 12176.99 & 9978.4 \\
\hline
\end{tabular}




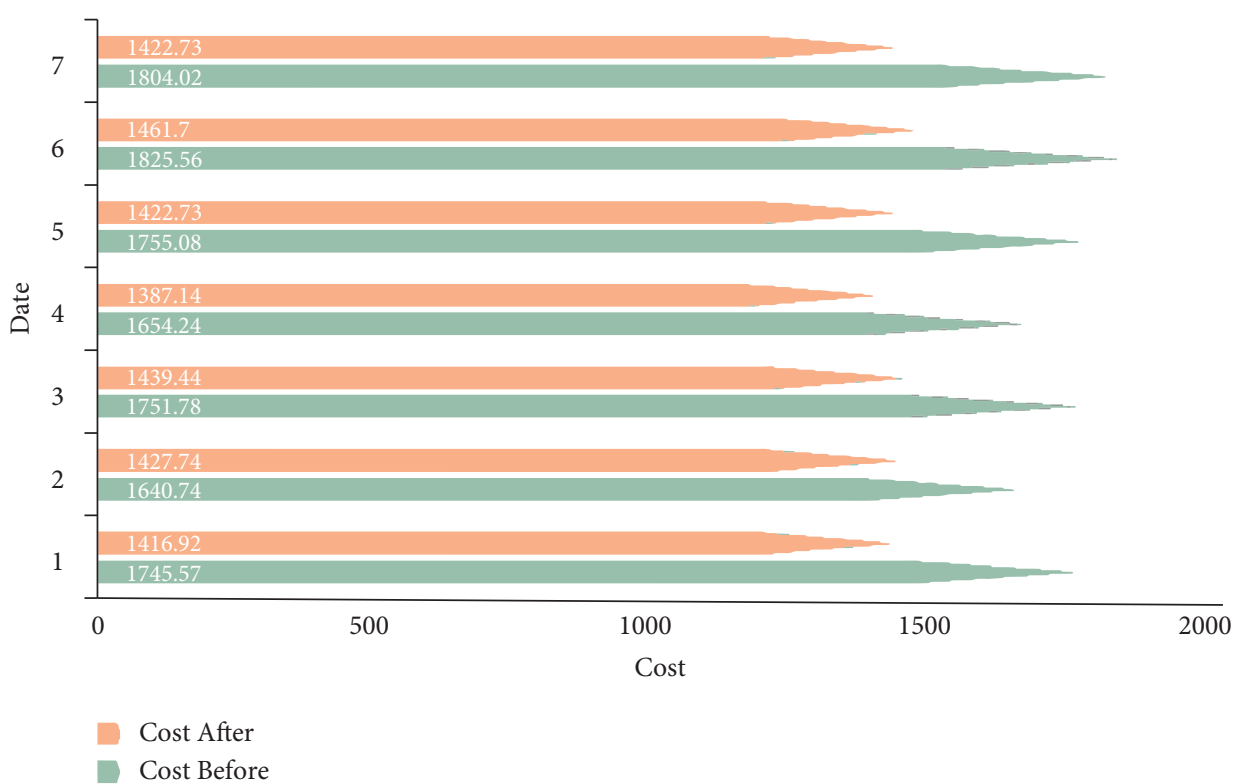

Figure 7: Comparison of daily distribution cost before and after model application.

TABLE 5: Comparison of distribution cost composition.

\begin{tabular}{lccc}
\hline Date & Fixed cost & Fuel consumption cost & Penalty cost \\
\hline 1 & 1200 & 533.65 & 11.92 \\
2 & 1200 & 425.43 & 15.31 \\
3 & 1200 & 538.62 & 13.16 \\
4 & 1200 & 435.84 & 18.4 \\
5 & 1200 & 534.58 & 20.5 \\
6 & 1200 & 601.3 & 24.26 \\
7 & 1200 & 588.32 & 15.7 \\
Total & 8400 & 3657.74 & 119.25 \\
\hline Date & Fixed cost & Fuel consumption cost & Penalty cost \\
1 & 1200 & 213.6 & 3.32 \\
2 & 1200 & 225.39 & 2.35 \\
3 & 1200 & 235.68 & 3.76 \\
4 & 1200 & 185.34 & 1.8 \\
5 & 1200 & 220.4 & 2.33 \\
6 & 1200 & 259.32 & 2.38 \\
7 & 1200 & 220.78 & 1.95 \\
Total & 8400 & 1560.51 & 17.89 \\
\hline
\end{tabular}

reduced by $85 \%$ due to efficient distribution. The cost structure of the model before application is analyzed.

As shown in Figure 8, the fixed cost accounts for the highest proportion of the daily cost before the application of the model, with an average of about $69 \%$. The average fuel consumption cost accounts for about $30 \%$, and the penalty cost accounts for about $1 \%$.

4.3.2. Satisfaction Survey. A reasonable questionnaire analysis is conducted on the satisfaction survey based on the survey model. After the model is run, a random survey is conducted on the employees, customers, and delivery drivers of the logistics company. The number of people participating in the survey is 5 (represented by numbers $1-5)$. The full score for satisfaction is 10 points. The lower the score, the lower the satisfaction. The specific scores are as follows.

As shown in Figure 9, all people's satisfaction scores were 9 or above, with an average score of 9.42. Two of the full scores were given by the company's employees and drivers, and the lowest score of 9 was scored by the company's employees and customers. Among the participants with three identities, the lowest average score of customers satisfaction was (9.12). This shows that the model has room for improvement in customer satisfaction. 


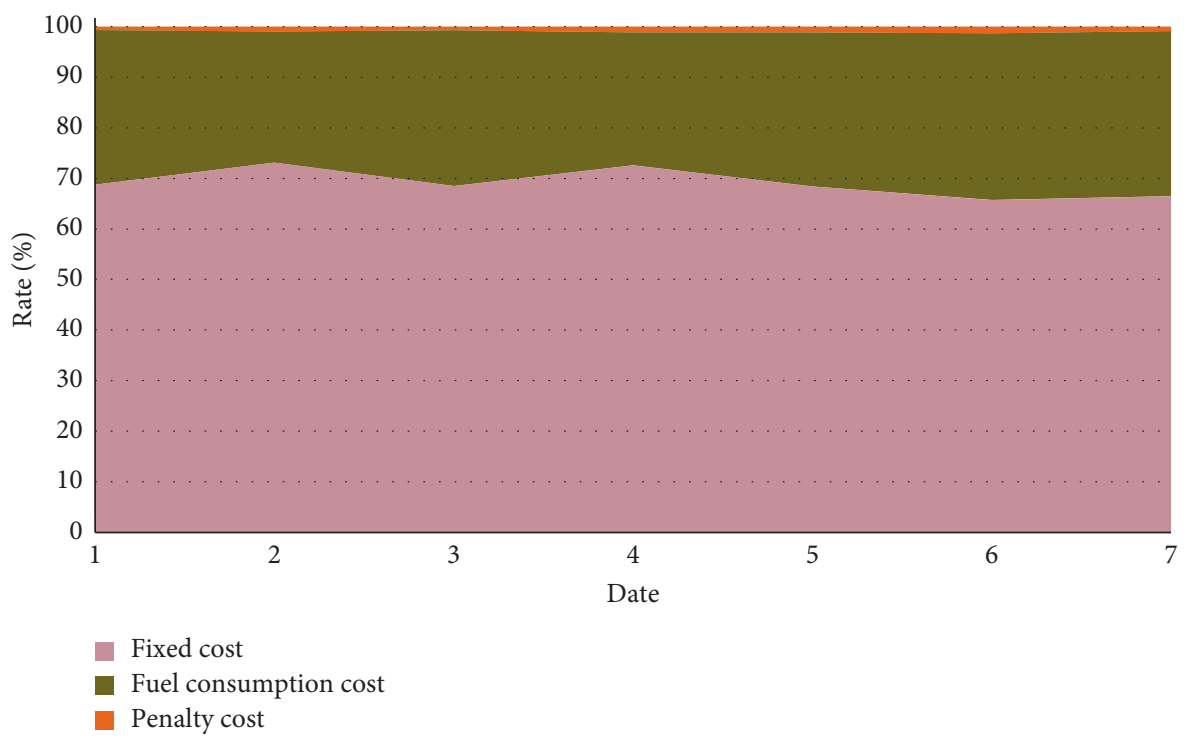

Figure 8: Composition of daily distribution cost before model application.

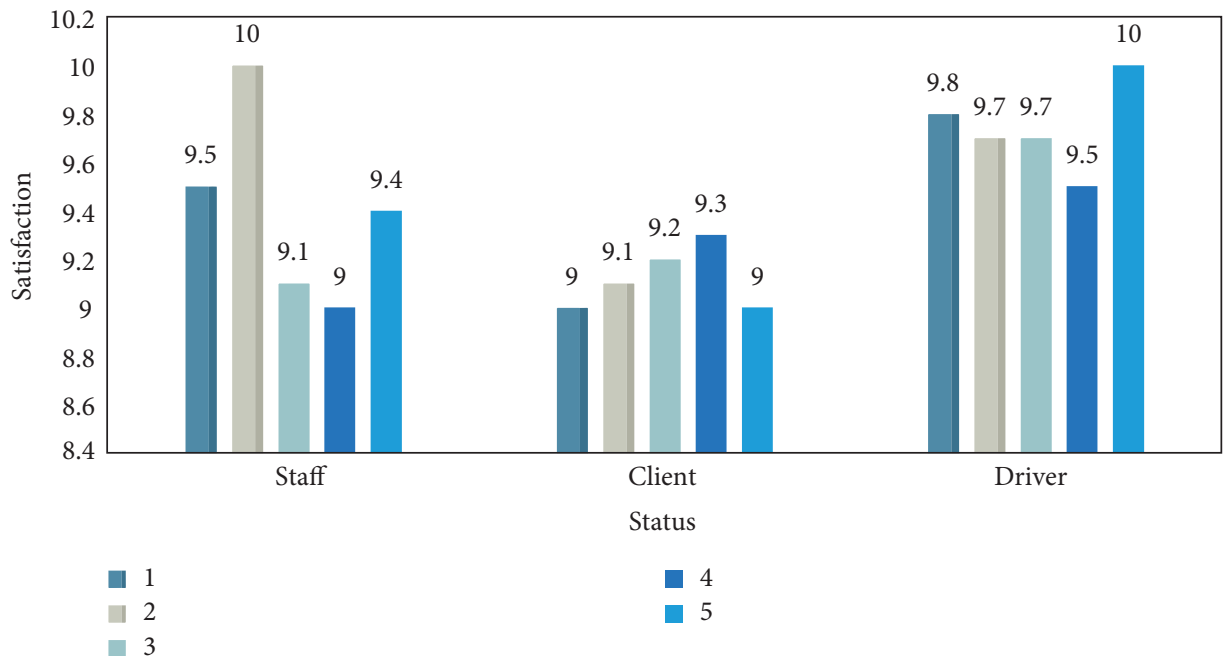

FIGURE 9: Satisfaction survey results.

\section{Conclusions}

The expressway service area is adjacent to the expressway, so it is convenient to pick up vehicles. It has the advantages of being fast and convenient in logistics transportation and distribution. The expressway service area can provide logistics services to the neighboring city nodes, produce a certain scale of logistics demand generation, and relay an attraction effect and logistics added value service, which has the advantages of logistics radiation effect. Using expressway to build logistics transportation channel can reduce the cost of logistics transportation, improve the efficiency of logistics transportation, promote the travel of comprehensive transportation system, and improve the capacity of regional transportation.

The LRP particle swarm optimization model of highway logistics network planning established in this paper has strong search ability and stability and can work out the optimal distribution scheme according to the optimal objective function value. In the actual distribution work, it can effectively save the distribution cost and improve the satisfaction. Therefore, this model can provide reference for solving LRP problem of expressway logistics network planning.

This paper only does some exploratory preliminary research on the LRP particle swarm optimization model and its algorithm in highway logistics network planning, but due to limited time and knowledge, a complete plan has not yet been formed. In future research work, it is necessary to further explore the cooperation between expressway logistics network and other modes of transportation, as well as the transformation of expressway service areas and distribution centers.

\section{Data Availability}

The data that support the findings of this study are available from the corresponding author upon reasonable request. 


\section{Conflicts of Interest}

The authors declared no potential conflicts of interest with respect to the research, authorship, and/or publication of this article.

\section{References}

[1] T. K. L. Hui, R. S. Sherratt, and D. D. Sanchez, "Major requirements for building smart homes in smart cities based on internet of things technologies," Future Generation Computer Systems, vol. 76, no. nov, pp. 358-369, 2016.

[2] C. F. Wang and Y. C. Chiu, "Routing problems for vehicle adhoc networks using the virtual message ferry routing scheme," International Journal of Ad Hoc and Ubiquitous Computing, vol. 28, no. 4, pp. 258-273, 2018.

[3] M. S. Balaji and S. K. Roy, "Value co-creation with Internet of things technology in the retail industry," Journal of Marketing Management, vol. 33, no. 1-2, pp. 7-31, 2017.

[4] D. Bo, D. Kangcheng, C. Guang, C. Huiyun, and Y. Hongying, "Carbon emission management system of port logistics based on internet of things technology," Agro Food Industry HiTech, vol. 28, no. 1, pp. 1094-1098, 2017.

[5] J. Guo, X. Wang, S. Fan, and M. Gen, "Forward and reverse logistics network and route planning under the environment of low-carbon emissions: a case study of Shanghai fresh food E-commerce enterprises," Computers \& Industrial Engineering, vol. 106, no. APR, pp. 351-360, 2017.

[6] Y. Hao and S. Wei, "An improved multi-objective programming with augmented $\varepsilon$-constraint method for hazardous waste location-routing problems," International Journal of Environmental Research \& Public Health, vol. 13, no. 6, p. 548, 2016.

[7] S. Panchanathan, S. Chakraborty, T. Mcdaniel et al., "Enriching the fan experience in a smart stadium using internet of things technologies," International Journal of Semantic Computing, vol. 11, no. 2, pp. 137-170, 2017.

[8] S. Zhao, S. Li, and Y. Yao, "Blockchain enabled industrial internet of things technology," IEEE Transactions on Computational Social Systems, vol. 6, no. 6, pp. 1442-1453, 2019.

[9] K.-C. Chang and Y. M. Seow, "Protective measures and security policy non-compliance intention," Journal of Organizational and End User Computing, vol. 31, no. 1, pp. 1-21, 2019.

[10] A. S. Deese and J. Daum, "Application of ZigBee-based internet of things technology to demand response in smart grids," IFAC-PapersOnLine, vol. 51, no. 28, pp. 43-48, 2018.

[11] Y. Zhang and J. Wen, "The IoT electric business model: using blockchain technology for the internet of things," Peer-to-Peer Networking and Applications, vol. 10, no. 4, pp. 983-994, 2017.

[12] M. Liu, J. Ma, L. Lin, M. Ge, Q. Wang, and C. Liu, "Intelligent assembly system for mechanical products and key technology based on internet of things," Journal of Intelligent Manufacturing, vol. 28, no. 2, pp. 271-299, 2017.

[13] T. K. L. Hui, R. S. Sherratt, and D. D. Sanchez, "Major requirements for building smart homes in smart cities based on internet of things technologies," Future Generation Computer Systems, vol. 76, pp. 358-369, 2016.

[14] G. W. Y. Wang, Q. Zeng, K. Li, and J. Yang, "Port connectivity in a logistic network: the case of Bohai Bay, China," Transportation Research Part E: Logistics and Transportation Review, vol. 95, pp. 341-354, 2016.
[15] A. T. Richter and S. Stiller, "Robust strategic route planning in logistics," Transportation Science, vol. 52, no. 1, pp. 38-58, 2018.

[16] J. Santagate, "Micrologistics as a strategy for improving your network," Supply chain brain, vol. 20, no. 1, p. 72, 2016.

[17] M. Schiffer and G. Walther, "Strategic planning of electric logistics fleet networks: a robust location-routing approach," Omega, vol. 80, pp. 31-42, 2018.

[18] D. Kannan, K. Garg, P. C. Jha, and A. Diabat, "Integrating disassembly line balancing in the planning of a reverse logistics network from the perspective of a third party provider," Annals of Operations Research, vol. 253, no. 1, pp. 353-376, 2017.

[19] M. R. Reddy, K. G. Srinivasa, and B. E. Reddy, "Smart vehicular system based on the internet of things," Journal of Organizational and End User Computing, vol. 30, no. 3, pp. 45-62, 2018.

[20] R. Wang, M. Z. Qi, and A. Q. Han, "Comparative study of emergency logistics path planning," Journal of Donghua University, vol. 35, no. 3, pp. 79-83, 2018.

[21] M. Rahimi and V. Ghezavati, "Sustainable multi-period reverse logistics network design and planning under uncertainty utilizing conditional value at risk $(\mathrm{CVaR})$ for recycling construction and demolition waste," Journal of Cleaner Production, vol. 172, no. 2, pp. 1567-1581, 2018.

[22] Z. Dai, F. Aqlan, K. Gao, and Y. Zhou, "A two-phase method for multi-echelon location-routing problems in supply chains," Expert Systems with Applications, vol. 115, pp. 618634, 2019.

[23] E. Fernández, G. Laporte, and J. Rodríguez-Pereira, "Exact solution of several families of location-arc routing problems," Transportation Science, vol. 53, no. 5, pp. 1313-1333, 2019.

[24] F. Glover and C. Rego, "New assignment-based neighborhoods for traveling salesman and routing problems," Networks, vol. 71, no. 3, pp. 170-187, 2018.

[25] Y. Karuno, H. Nagamochi, and A. Shurbevski, "Constant factor approximation algorithms for repetitive routing problems of grasp-and-delivery robots in production of printed circuit boards," Journal of the Operations Research Society of Japan, vol. 55, no. 3, pp. 181-191, 2017.

[26] I. S. Litvinchev, G. Cedillo, and M. Velarde, "Integrating territory design and routing problems," Journal of Computer and Systems Sciences International, vol. 56, no. 6, pp. 969-974, 2017. 